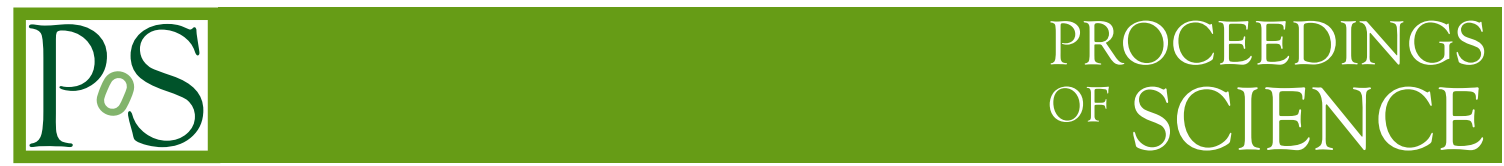

\title{
Electromagnetic Follow-up of GW150914
}

\author{
Mario C. Díaz ${ }^{* \dagger}$ \\ The University of Texas Rio Grande Valley \\ E-mail: mario.diaz@utrgv.edu
}

\begin{abstract}
This is a review of the LIGO first gravitational wave detections announced during 2016 and the signification they have as the birth of gravitational wave astronomy. We briefly describe the results, some of it possible astrophysical implications, and also report about electromagnetic followup efforts during the first detection.
\end{abstract}

XII Multifrequency Behaviour of High Energy Cosmic Sources Workshop

12-17 June

Palermo, Italy

\footnotetext{
*Speaker.

${ }^{\dagger}$ On behalf of the TOROS Collaboration
} 


\section{Introduction}

On September 14, 2015 at 09:50:45 UTC, the LIGO observatories in Hanford, Washington, and Livingston, Louisiana, both in the USA, detected a gravitational wave: GW150914 [5]. The signal was observed with a signal to noise ratio of 24 (using matched filtering techniques) and a false alarm rate lower than 1 event in 203.000 years, equivalent to a $5.1 \sigma$ significance. Since the formulation of the General Theory of Relativity in 1915 [18] and the prediction of gravitational waves $(\mathrm{GW})[19]$ one hundred years have gone by until their detection. This is a history with plenty of frustrations and confusion. The transformational event is the result of both the maturity in the development of the required technologies and the astrophysical and theoretical developments over the last several years which made it possible. Following the uncertainties and doubts that affected the theoretical work advanced by the scientific community for almost fifty years, the last stretch of the XX century and the beginning of this one was witness to many experimental and theoretical breakthroughs that were crucial to lead to this discovery. The work by Joseph Weber [37] motivated a new generation of experimental physicists to try new technologies and build confidence in the feasibility of making a first detection as a realistic and doable endeavor. Hulse and Taylor observations of the binary pulsar PSR 1913+16 [24] [35] showed the existence of compact sources loosing gravitational energy at the rate consistent with the emission of gravitational radiation. And more recently the computational work of a new generation of numerical relativity scientists bear fruits achieving the first realistic models simulating binary black hole mergers [32], [14], [9]. A relevant aspect of the scientific merit of this first detection from the LIGO observatories is that it demonstrates the existence of binary black hole systems with stellar masses. It also constitutes the first direct detection of gravitational waves and the first observation of a binary black hole merger. A second detection was made on December 26, 2016 [3]. Also in this case the signal observed corresponded to the merger of a binary black hole system. In the remaining of this paper the main characteristics of the systems detected will be presented and its astrophysical relevance discussed, particularly in the context of stellar evolution theory. The joint effort of many observational groups that participated in the follow-up efforts to observe the possible existence of electromagnetic counterparts to the sources observed by LIGO will also be described, and in particular the follow-up efforts by the TOROS collaboration in Argentina.

\section{Some aspects of the first detections and their astrophysical implications}

GW150914 was detected after several hours of continued operation before and after the event. The susceptibility to environmental perturbations was measured through the detection of several induced predetermined excitations, of magnetic, radio-frequency, acoustic and other vibrational characteristics. The environmental sensors did not register perturbations during the detection. Environmental fluctuations constituted only about $6 \%$ of the stress amplitude (main channel) during the event. Similarly there were no temporal correlations of instrumental perturbations between the two detectors. A total of 16 days of coincidental observations by the two detectors between September 12 and October 20, 2015 were used to assess the significance of the GW150914 event, through two independent analyses. One is optimized to detect binary compact systems coalescence utilizing optical matched filtering techniques using templates provided by general relativity theory 
and post-newtonian approximations ([5] and references therein). The other method searches for a range of transient signals with minimal assumptions about the waveforms (burst pipeline). A statistical significance is assigned to the candidate events according to the probability that they could be a gravitational wave. The relevance of the found candidate events is estimated by a background search identifying the rate at which the detector noise is producing events with a statistical significance equal or larger than these candidates. The background is estimated differently for the two searches, but both utilize a time shift technique: the values of each detector data are displaced by a time larger than the light propagation time between both sites and a new set of events is produced with this shift. If a signal in one detector coincides with the time shifts in the other this adds to the background estimation, which conducts to an overestimation of the background and a more conservative estimate of the candidates significance.

GW150914 is found with a significance of $4.6 \sigma$ in the burst case and larger than $5.1 \sigma$ with the matched filtering method. The physical parameters of GW150914 identified the merger of a black hole $(\mathrm{BH})$ of $36_{-4}^{+5} M_{\odot}$ and another one of $29_{-4}^{+4} M_{\odot}$ resulting in a final $\mathrm{BH}$ of $62_{-4}^{+4} M_{\odot}$. The GW151226 event, although found with a probability larger than $5 \sigma$ with the matched filtering method, was found with a signal to noise ratio of only 13 (almost half of the one for GW150914). The physical parameters for GW151226 indicate that a BH of $14.2_{-3.7}^{+8.3} M_{\odot}$ merged with another one of $7.5_{-2.3}^{+2.3} M_{\odot}$ resulting in a final BH of $20.8_{-1.7}^{+6.1} M_{\odot}$. We can can compare the astrophysical significance of these results with the BHs observed until now. Cygnus X-1, the first BH found and clearly established as such was discovered in 1972. Dynamical observations of its companion star (a massive supergiant $B 0$ ) showed that the compact X-ray source had a mass larger than those accepted for neutron stars and must then be a BH. In 1986 a new class of BHs in binary systems was found with different characteristics: the optical companion is a star of low mass and the X-ray source oscillates between periods of explosive activity -which can last up to a year- and periods of long quietness. There are several sources of this kind, systems that emit X-rays and contain a source of a mass that is estimated to be a BH. All these BHs are generically denominated X-ray Binaries (XRB) [27]. 22 XRB BHs with dynamical measurements of their masses have been confirmed with 19 of them in our galaxy. The majority of them have $\mathrm{BH}$ masses between $5-10 M_{\odot}$, while other have masses in the range of $10-20 M_{\odot}$ [21]. All these systems have been observed in low density stellar zones. The BHs associated to GW150914 and GW151226 are more massive than the BHS associated to XRBs (Fig. 1).

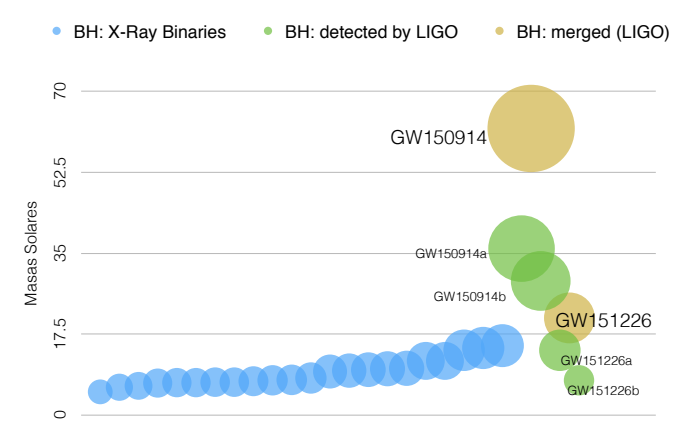

Figure 1: Observed Black Holes during O1

The BH resulting from the coalescence behind GW150914 provides a clear evidence of the 
existence of "heavy" BHs (masses larger than $25 M_{\odot}$ ). This discovery suggests the existence of relatively weak massive stellar winds indicating that possibly GW150914 was formed in an environment with metallicities lower than half of the solar value. This observation is also consistent with the models that predict larger formation values ( $\left.\gtrsim 1 G p c^{-3} y r^{-1}\right)$. The low GW150914 redshift $(z \simeq 0.1)$ and the inference of a low metallicity stellar progenitor implies two possibilities: either binary BHs are produced in galactic systems of low mass in the local universe and experiment an early merger or these systems are formed at larger redshifts with a delay from formation to merger of several Gyr [2].

\section{Electromagnetic follow-up}

The network of advanced gravitational wave interferometers, formed by LIGO [26], which started operations on September 2015, and the VIRGO observatory [8], which started operations in 2017, have been designed with the capability of detecting GWs emitted by the fusion of binary neutron stars (BNS) and black holes in binary systems $(\mathrm{BBH})$ at a distance of hundreds of Mpc [6]. Anticipating the operation of such a network on June 6, 2013 the LIGO-VIRGO collaboration called $^{1}$ the world astronomical community to participate in the multimessenger observation of astrophysical events observed by the GW detectors, using a vast range of telescopes and instruments from "traditional" astronomy. During the first detections the alarms to be sent, announcing the observation of possible candidates, would have been shared only with observer groups that have signed Memorandum of Understanding (MOU) with the LVC involving an agreement over the scientific products and information with the observations to be made. It was expected that the fusion of binary compact systems with at least one neutron star would have an associated electromagnetic radiation produced during the event. This electromagnetic (EM) counterpart originated in the interaction of the ejected material with a medium rich in neutrons could span from Gamma Ray Bursts (GRBs) of short duration to optical emission including the near infrared (so called kilonovae) and reach also radio wavelengths [25], [29], [28], [10], [13], [16]. The simultaneous detection of an event of such characteristics by both the GW and EM observatories (including the neutrino and cosmic ray observatories) could lead to a more comprehensive astrophysical interpretation of the event and better estimates of the distance and energy scale of it. The follow-up efforts from the different groups that signed MOUs with the LVC was reported in [4] and [7].

\section{Observations by the TOROS collaboration}

The TOROS scientific collaboration was formed to participate in the follow-up of LIGOVIRGO alerts. TOROS stands for "Transient Optical Robotic Observatory of the South" (TOROS) [12], and its main objective is to install a wide field of view telescope in the Atacama plateau, in the Argentine Northwest [33], [36]. The collaboration decided to utilize other resources independently of the construction of this facility. On April 5, 2014, TOROS signed an MoU with LVC and participated in the first observational run (O1) of the LIGO interferometers from September 2015 through January of 2016. Two facilities were available during O1: a 0.4m Schmidt-Cassegrain in Cordón

\footnotetext{
${ }^{1}$ http://www.ligo.org/scientists/GWEMalerts.php
} 


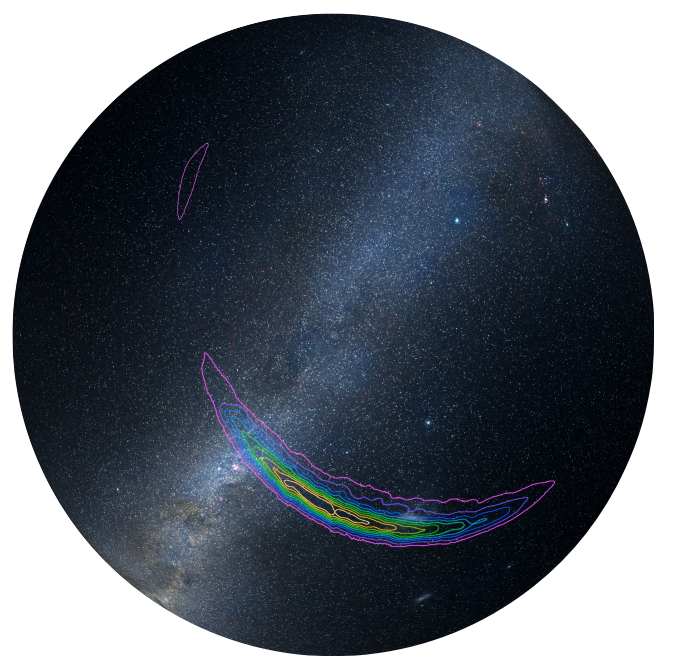

Figure 2: approximate location of GW150914, image credit: LIGO/Axel Mellinger

Macón and the 1,5m telescope of the Estación Astrofísica Bosque Alegre (EABA) in Córdoba, Argentina. The LVC provided two maps indicating the probability of localization of the GW150914 source in the sky. These maps, "coherent Wave Burst" and the "Omicron+LALInference Burst" (see [5]) were optimized to find signals without any prior modeling. The first one, a rapid localization analysis explores the possible existence of a power excess in both detectors and the second one searches for a sine-gaussian signal. These maps provide credible regions with an initial localization probability of 50\% and 90\%, covering about 200 and 750 square degrees respectively ( [34]).

TOROS started observation immediately after receiving the GCN informing about the detection event later denominated GW150914, and secured a first epoch of observations on 2015 September 16 and 17. A second epoch of images was obtained on December 5 and 6 of 2015 to be used as references in the differential photometric analysis. An Apogee Alta U9 camera was utilized with a field of vision ( FoV) of $12^{\prime} 7 \times 8^{\prime} 5$ and an effective plate scale of $0^{\prime} 75 \mathrm{pix}^{-1}$ after applying a $3 \times 3$ binning. To maximize sensitivity observations without filter were performed covering a range of $0.35<\lambda / \mu m<1.60 \mathrm{~s}$ individual exposures were obtained while the "seeing" had a median (FWHM) of 2.8". 10 images per field were obtained reaching limiting magnitudes of $r=21.7 \pm 0.3$ mag with $5 \sigma$ [17]. LIGO localization regions extend over hundred of square degrees (see Fig. 2) and vary depending of the algorithm used in calculating them. For example, an area of $90 \%$ credible localization probability for the cWB algorithm, covers 310 square degrees while other localization algorithms extend up to 750 square degrees (see table 1 in [7]). Independently of these maps properties, all of them are consistent with a wide and long arch in the southern hemisphere with a very small extension in the northern hemisphere. The cWB algorithm provides certain maps for binary BHs signals but underestimates the extension to the regions of high reliability [20]. In Fig. 2 the observed galaxies are shown (See Table 1). As it can be seen in the Fig. 3, the adoption of alternative maps not available at the time of the follow-up observations were performed, reduced significantly the regions with high probability observed by TOROS' small FoV.

Previous publications ([30], [1], [23]) have shown that the utilization of galactic maps increases the probability of finding an electromagnetic counterpart in the case of binary compact 
Table 1: Galaxies observed

\begin{tabular}{lcc}
\hline \hline Date & $I D$ & $D(M p c)$ \\
\hline $2015-09-16$ & IC1933 & 17.45 \\
$2015-09-16$ & NGC1529 & 54.76 \\
$2015-09-16$ & IC2038 & 7.00 \\
$2015-09-16$ & IC2039 & 7.63 \\
$2015-09-17$ & ESO058-018 & 52.23 \\
$2015-09-17$ & ESO084-015 & 14.99 \\
$2015-09-17$ & ESO119-005 & 9.73 \\
$2015-09-17$ & NGC1559 & 12.59 \\
$2015-09-17$ & PGC016318 & 9.54 \\
$2015-09-17$ & PGC269445 & 54.83 \\
$2015-09-17$ & PGC280995 & 55.08 \\
$2015-09-17$ & PGC128075 & 63.71 \\
$2015-09-17$ & PGC381152 & 13.26 \\
$2015-09-17$ & PGC075108 & 13.29
\end{tabular}
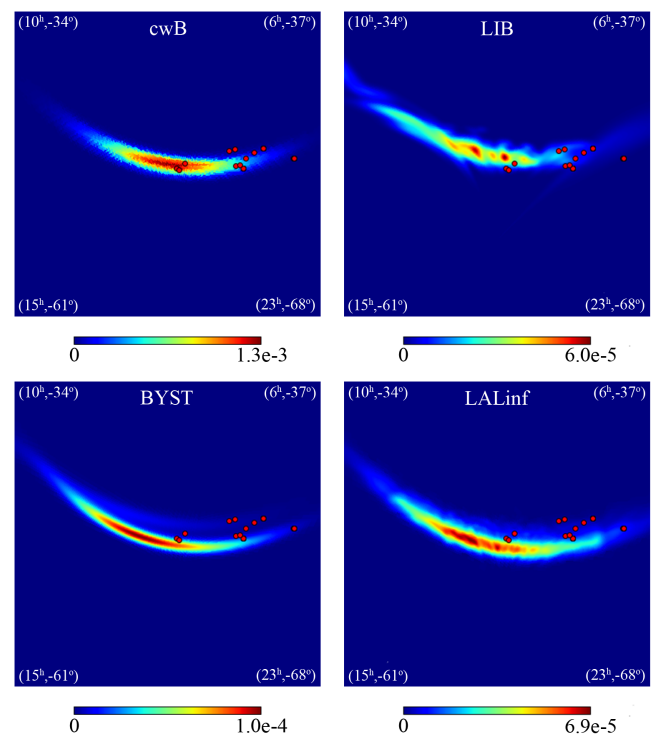

Figure 3: localization probability maps of GW150914 generated by the different LVC algorithms, indicating with red dots the localization of the galaxies observed see [17]

mergers where at least one of the objects is a neutron star. TOROS optimized its analysis for the small FoV selecting for observation nearby galaxies with the largest probability of hosting the event. The probabilities were based on the pixel values in the initial cWB map containing the coordinates of a specific galaxy. The GW galactic catalog was utilized. The GWGC (GWGC), [38], is a compilation of catalogs homogenized to a list of $\sim 53.000$ galaxies within $100 \mathrm{Mpc}$ ( incomplete starting at $D \sim 40 \mathrm{Mpc}$ ). Table 1 lists the galaxies observed following this criterion. The GWGC provides distances, blue magnitudes and other properties. The selection was performed following 
an ad hoc "calendar" (a Python module in the TOROS pipeline ). The calendar list of criteria were: (1) visibility from observing location ( $\left.30^{\circ}>\delta>-70^{\circ}\right)$, (2) apparent magnitude $B \leq 21 \mathrm{mag}$, and (3) distance $D<60 \mathrm{Mpc}$. The cut in magnitude is motivated by the expectation that in the near universe, the distribution of binary neutron stars and BHs, due to the short duration of the fusion time scale must follow recent star formation [31], [11]. Once a relationship between sky maps and galaxies is established according to the applied filters, a probability ranking $P_{g, i}$ is established (where $i$ refers to the skymap pixel containing the galaxy $g$ ). The different observational targets receive then a prioritization according to the localization in the maps and their visibility. A final requirement consists in observing the targets within a field of size $\sim 5 \mathrm{kpc}$, corresponding to the median separation between short duration GRBs and their host galaxies, observed after optical afterglows [15], [22], [13]. This requirement forced telescope operators to cover some objectives with more than one image. A total of 21 fields were observed covering 14 galaxies. These correspond to $\sim 4.4 \%$ of the host galaxies that could have been observed following the above criteria and included in the GWGC. The catalog is considered complete up to $80 \%$ up to $60 \mathrm{Mpc}$-criterion 2[38]. The obtained observations were analyzed by two different implementation of differentiation algorithms [17]. After the image processing a "Random-Forest" algorithm was utilized to discriminate between real and bogus candidates. No significant event was found in the surveyed area up to a limiting magnitude of $r=21.7$ mag $(\mathrm{AB})$ with a $5 \sigma$ certainty.

\section{Conclusion}

An analysis of the first GW detections made by the LVC during $\mathrm{O} 1$ was presented and the significance they have for the new nascent science of gravitational wave astronomy was made. The results of the astrophysical implications of these observations as well as some of the characteristics of the electromagnetic follow-up efforts conducted were also discussed. The particular efforts of the TOROS collaboration were presented and discussed. Its results are consistent with the LIGO detection of a BBH merger for which no electromagnetic counterpart was expected. The TOROS observations (were no transient event was observed) are also consistent with the rate of transient events expected. The relevance of these observations consists in showing the possibility of utilizing small FoV instruments for follow-up activities.

\section{References}

[1] J. Abadie, B. P. Abbott, R. Abbott, T. D. Abbott, M. Abernathy, T. Accadia, F. Acernese, C. Adams, R. Adhikari, C. Affeldt, and et al. First low-latency LIGO+Virgo search for binary inspirals and their electromagnetic counterparts. A\&A, 541:A155, May 2012.

[2] B. P. Abbott, R. Abbott, T. D. Abbott, M. R. Abernathy, F. Acernese, K. Ackley, C. Adams, T. Adams, P. Addesso, R. X. Adhikari, and et al. Astrophysical Implications of the Binary Black-hole Merger GW150914. ApJL, 818:L22, February 2016.

[3] B. P. Abbott, R. Abbott, T. D. Abbott, M. R. Abernathy, F. Acernese, K. Ackley, C. Adams, T. Adams, P. Addesso, R. X. Adhikari, and et al. GW151226: Observation of Gravitational Waves from a 22-Solar-Mass Binary Black Hole Coalescence. Physical Review Letters, 116(24):241103, June 2016. 
[4] B. P. Abbott, R. Abbott, T. D. Abbott, M. R. Abernathy, F. Acernese, K. Ackley, C. Adams, T. Adams, P. Addesso, R. X. Adhikari, and et al. Localization and Broadband Follow-up of the Gravitational-wave Transient GW150914. ApJL, 826(1):L13, July 2016.

[5] B. P. Abbott, R. Abbott, T. D. Abbott, M. R. Abernathy, F. Acernese, K. Ackley, C. Adams, T. Adams, P. Addesso, R. X. Adhikari, and et al. Observation of Gravitational Waves from a Binary Black Hole Merger. Physical Review Letters, 116(6):061102, February 2016.

[6] B. P. Abbott, R. Abbott, T. D. Abbott, M. R. Abernathy, F. Acernese, K. Ackley, C. Adams, T. Adams, P. Addesso, R. X. Adhikari, and et al. Prospects for Observing and Localizing Gravitational-Wave Transients with Advanced LIGO and Advanced Virgo. Living Reviews in Relativity, 19, February 2016.

[7] B. P. Abbott, R. Abbott, T. D. Abbott, M. R. Abernathy, F. Acernese, K. Ackley, C. Adams, T. Adams, P. Addesso, R. X. Adhikari, and et al. Supplement: Localization and broadband follow-up of the gravitational-wave transient GW150914. Astrophysical Journal Supplement Series, 225(1):8, July 2016.

[8] F. Acernese, M. Agathos, K. Agatsuma, D. Aisa, N. Allemandou, A. Allocca, J. Amarni, P. Astone, G. Balestri, G. Ballardin, and et al. Advanced Virgo: a second-generation interferometric gravitational wave detector. Classical and Quantum Gravity, 32(2):024001, January 2015.

[9] J. G. Baker, J. Centrella, D.-I. Choi, M. Koppitz, and J. van Meter. Gravitational-Wave Extraction from an Inspiraling Configuration of Merging Black Holes. Physical Review Letters, 96(11):111102, March 2006.

[10] J. Barnes and D. Kasen. Effect of a High Opacity on the Light Curves of Radioactively Powered Transients from Compact Object Mergers. ApJ, 775:18, September 2013.

[11] K. Belczynski, V. Kalogera, and T. Bulik. A Comprehensive Study of Binary Compact Objects as Gravitational Wave Sources: Evolutionary Channels, Rates, and Physical Properties. ApJ, 572:407-431, June 2002.

[12] M. Benacquista, C. Belczynski, M. Beroiz, M. Branchesi, C. Colazo, M. C. Diaz, M. Dominguez, D. Garcia Lambas, S. Liang, L. Macri, T. Peñuela, B. Sanchez, M. Schneiter, and C. V. Torres. A program for optical observations of advanced LIGO early triggers in the southern hemisphere. In EAS Publications Series, volume 67 of EAS Publications Series, pages 357-358, July 2014.

[13] E. Berger. Short-Duration Gamma-Ray Bursts. Annual Review of Astronomy and Astrophysics, 52:43-105, August 2014.

[14] M. Campanelli, C. O. Lousto, P. Marronetti, and Y. Zlochower. Accurate Evolutions of Orbiting Black-Hole Binaries without Excision. Physical Review Letters, 96(11):111101, March 2006.

[15] R. P. Church, A. J. Levan, M. B. Davies, and N. Tanvir. Implications for the origin of short gamma-ray bursts from their observed positions around their host galaxies. MNRAS, 413:2004-2014, May 2011.

[16] P. S. Cowperthwaite and E. Berger. A Comprehensive Study of Detectability and Contamination in Deep Rapid Optical Searches for Gravitational Wave Counterparts. ApJ, 814:25, November 2015.

[17] M. C. Díaz, M. Beroiz, T. Peñuela, L. M. Macri, R. J. Oelkers, W. Yuan, D. García Lambas, J. Cabral, C. Colazo, M. Domínguez, B. Sánchez, and S. Gurovich. GW150914: First Search for the Electromagnetic Counterpart of a Gravitational-wave Event by the TOROS Collaboration. ApJL, 828:L16, September 2016.

[18] A. Einstein. The field equations of gravitation. Königlich Preußische Akademie der Wissenschaften (Berlin). Sitzungsberichte (1915), pages 844-847, December 1915. 
[19] A Einstein and K. Sitzungsber. . Preuss. Akad. Wiss., page 688, December 1916.

[20] R. Essick, S. Vitale, E. Katsavounidis, G. Vedovato, and S. Klimenko. Localization of Short Duration Gravitational-wave Transients with the Early Advanced LIGO and Virgo Detectors. ApJ, 800:81, February 2015.

[21] W. M. Farr, N. Sravan, A. Cantrell, L. Kreidberg, C. D. Bailyn, I. Mandel, and V. Kalogera. The Mass Distribution of Stellar-mass Black Holes. ApJ, 741:103, November 2011.

[22] W. Fong and E. Berger. The Locations of Short Gamma-Ray Bursts as Evidence for Compact Object Binary Progenitors. ApJ, 776:18, October 2013.

[23] C. Hanna, I. Mandel, and W. Vousden. Utility of Galaxy Catalogs for Following up Gravitational Waves from Binary Neutron Star Mergers with Wide-field Telescopes. ApJ, 784:8, March 2014.

[24] R. A. Hulse and J. H. Taylor. Discovery of a pulsar in a binary system. ApJL, 195:L51-L53, January 1975.

[25] L.-X. Li and B. Paczyński. Transient Events from Neutron Star Mergers. ApJL, 507:L59-L62, November 1998.

[26] LIGO Scientific Collaboration, J. Aasi, B. P. Abbott, R. Abbott, T. Abbott, M. R. Abernathy, K. Ackley, C. Adams, T. Adams, P. Addesso, and et al. Advanced LIGO. Classical and Quantum Gravity, 32(7):074001, April 2015.

[27] J. McClintock. Black Hole X-ray Binaries. In APS April Meeting Abstracts, May 2004.

[28] B. D. Metzger and E. Berger. What is the Most Promising Electromagnetic Counterpart of a Neutron Star Binary Merger? ApJ, 746:48, February 2012.

[29] E. Nakar and T. Piran. Detectable radio flares following gravitational waves from mergers of binary neutron stars. Nature, 478:82-84, October 2011.

[30] L. K. Nuttall and P. J. Sutton. Identifying the host galaxy of gravitational wave signals. Physical Review D, 82(10):102002, November 2010.

[31] E. S. Phinney. The rate of neutron star binary mergers in the universe - Minimal predictions for gravity wave detectors. ApJL, 380:L17-L21, October 1991.

[32] F. Pretorius. Evolution of Binary Black-Hole Spacetimes. Physical Review Letters, 95(12):121101, September 2005.

[33] V. Renzi, R. Vrech, D. Ferreiro, D. García Lambas, M. Solinas, H. Muriel, J. Viramonte, M. Sarazin, and P. Recabarren. Caracterización astronómica del sitio Cordón Macón en la provincia de Salta. Boletin de la Asociacion Argentina de Astronomia La Plata Argentina, 52:285-288, 2009.

[34] L. Singer. LIGO/Virgo G184098: Burst candidate in LIGO engineering run data. GRB Coordinates Network, 18330, 2015.

[35] J. H. Taylor and J. M. Weisberg. A new test of general relativity - Gravitational radiation and the binary pulsar PSR 1913+16. ApJ, 253:908-920, February 1982.

[36] P. Tremblin, N. Schneider, V. Minier, G. A. Durand, and J. Urban. Worldwide site comparison for submillimetre astronomy. Astron. Astrophys, 548:A65, December 2012.

[37] J. Weber. Gravitational Radiation. Physical Review Letters, 18:498-501, March 1967.

[38] D. J. White, E. J. Daw, and V. S. Dhillon. A list of galaxies for gravitational wave searches. Classical and Quantum Gravity, 28(8):085016, April 2011. 